\title{
Assessment of Serum Nitrogen Species and Inflammatory Parameters in Relapsing-Remitting Multiple Sclerosis Patients Treated with Different Therapeutic Approaches
}

\author{
Natalia Niedziela, ${ }^{1}$ Monika Adamczyk-Sowa, ${ }^{1}$ Jacek T. Niedziela, ${ }^{2}$ Bogdan Mazur, ${ }^{3}$ \\ Ewa Kluczewska, ${ }^{4}$ Pawel Sowa, ${ }^{5}$ and Mariusz Gąsior ${ }^{2}$ \\ ${ }^{1}$ Department of Neurology in Zabrze, Medical University of Silesia, ul. 3-go Maja 13-15, 41-800 Zabrze, Poland \\ ${ }^{2} 3 r d$ Department of Cardiology, SMDZ in Zabrze, Medical University of Silesia, Silesian Centre for Heart Disease, \\ ul. Curie-Skłodowskiej 9, 41-800 Zabrze, Poland \\ ${ }^{3}$ Department of Microbiology and Immunology in Zabrze, Medical University of Silesia, ul. Jordana 19, 41-808 Zabrze, Poland \\ ${ }^{4}$ Department of Radiology in Zabrze, Medical University of Silesia, ul. 3-go Maja 13-15, 41-800 Zabrze, Poland \\ ${ }^{5}$ Department of Laryngology in Zabrze, Medical University of Silesia, ul. Curie-Skłodowskiej 10, 41-800 Zabrze, Poland
}

Correspondence should be addressed to Natalia Niedziela; natalia@niedziela.org

Received 12 July 2016; Revised 20 October 2016; Accepted 8 November 2016

Academic Editor: Markus Kipp

Copyright (C) 2016 Natalia Niedziela et al. This is an open access article distributed under the Creative Commons Attribution License, which permits unrestricted use, distribution, and reproduction in any medium, provided the original work is properly cited.

\begin{abstract}
The role of nitric oxide and its reactive derivatives $\left(\mathrm{NO}_{x}\right)$ is well known in the pathogenesis of multiple sclerosis, which is an inflammatory disease while $\mathrm{NO}_{x}$ seems to be important in coordinating inflammatory response. The purpose of the present study was to assess serum $\mathrm{NO}_{x}$ as one of the nitrogen species and inflammatory parameters in relapsing-remitting multiple sclerosis patients and to compare the effectiveness of various types of disease-modifying therapies that reduce nitric oxide and inflammatory biomarkers. Elevated $\mathrm{NO}_{x}$ level was observed in patients who received the first-line disease-modifying therapy (interferons beta-la and beta-1b) in comparison with the subjects treated with the second-line disease-modifying therapy (natalizumab; fingolimod) and healthy controls without significant differences in C-reactive protein and interleukin-1 beta. A negative correlation was observed between serum $\mathrm{NO}_{x}$ level and the duration of multiple sclerosis confirmed in the whole study population and in subjects treated with the first-line agents. Only serum $\mathrm{NO}_{x}$, concentration could reveal a potential efficacy of disease-modifying therapy with a better reduction in $\mathrm{NO}_{x}$ level due to the second-line agents of disease-modifying therapy.
\end{abstract}

\section{Introduction}

Multiple sclerosis (MS) is a chronic demyelinating and inflammatory disorder of the central nervous system (CNS) affecting approximately 2.5 million people worldwide $[1,2]$. It is characterized by immune-mediated cellular damage in the white and grey matters [3]. The etiology of MS remains unclear although both genetic and environmental factors are involved in disease onset and development $[4,5]$. The hallmark feature of MS lesion is the destruction of the myelin sheath, followed by formation of demyelinating plaques and loss of axons considered to be a marker of disease progression $[6,7]$.
The disease is related to a $\mathrm{T}$ cell-dependent process which is associated with macrophage-mediated demyelination due to myelin-specific autoantigens. Additionally, many critical players are involved in the pathogenesis of MS, including different immune cells, such as T helper lymphocytes (Th1 and Th17), B cells, or several autoantibodies directed against CNS components [8]. Meanwhile, infiltration of lymphocytes and monocytes from the peripheral blood into the brain is observed in acute active MS lesions [9]. According to some studies, immune-mediated myelin loss and mitochondrial dysfunction seem to play a crucial role in MS $[10,11]$. Different factors including oxidative stress cause mitochondrial destruction [12-14]. As a result, mitochondria are the main 
source of reactive oxygen species (ROS) and reactive nitrogen species (RNS) $[15,16]$. In the case of mitochondrial dysfunction, overproduction of toxic reactive species is observed. It plays a pivotal function in myelin and oligodendrocyte loss. They are many various types of RNS involved in the pathophysiology of MS, most of which are unstable due to their short lifetime. Additionally, most RNS are difficult to detect due to coexistence of many antioxidants in vivo. The role of nitric oxide (NO) and its reactive derivative (peroxynitrite) is well documented in the pathogenesis of MS $[17,18]$. Nitric oxide is unstable and is rapidly converted to nitrates and nitrites which reflect NO concentrations [19]. In MS the engagement of $\mathrm{NO}$ is related to the presence of peroxynitrite activities, $\mathrm{NO}$ metabolites, and inducible NO synthase (iNOS) [20]. Moreover, recent studies report dramatically increased oxidative stress in MS patients [5, 2123 ], including the occurrence of oxidative protein modifications $[24,25]$. Furthermore, reduction in antioxidant defense mechanism is involved in the pathogenesis of MS [26, 27].

It is well known that MS is an inflammatory disease while $\mathrm{NO}_{x}$ seems to be important in coordinating inflammatory response. In the course of MS, activated T cells and macrophages secrete proinflammatory cytokines such as interleukins (IL-1 and IL-12), interferon gamma (IFN$\gamma$ ), or tumor necrosis factor alpha (TNF- $\alpha$ ) [28]. These cytokines can enhance NO formation in the CNS by iNOS [29]. Recent studies have suggested that ROS and RNS lead to oxidative and nitrosative stress responsible for neurodegeneration. However, the association between neuroinflammation, nitrosative, and oxidative stress in MS is still poorly understood. It is still not fully understood which process initiates MS, the disease course or nitrosative and oxidative stress which occurs as a consequence of an inflammatory cascade [30]. Over the past few years many new therapeutic options have been involved in the treatment of MS. The current range of disease-modifying therapy (DMT) includes intervention in antigen presentation and peripheral immune response and in the blood-brain barrier [31]. The first-line agents of DMT [interferons beta (INF- $\beta 1 \mathrm{a} ; \beta-1 \mathrm{~b})$ and glatiramer acetate $(\mathrm{GA})]$ decrease proinflammatory cytokines and prevent the migration of activated $\mathrm{T}$ cells across the bloodbrain barrier [32]. Natalizumab (NT), the second-line agent of DMT, prevents such migration [33] while fingolimod (FG) prevents the emergence of lymphocytes from the lymphoid tissue decreasing lymphocyte infiltration into the CNS [34]. Additionally, FG acts directly on oligodendrocytes, microglia, astrocytes, and neurons [35]. It should be borne in mind that shift from inflammation to neurodegeneration is a significant feature of MS. Both immediate intervention to control inflammation and a delay in the progression of disability are required [36].

The aim of the study was to assess serum nitric oxide as one of the nitrogen species and inflammatory parameters in relapsing-remitting MS (RRMS) and to compare the effectiveness of various types of therapy (first- and secondline agents of DMT) to reduce nitric oxide and inflammatory biomarkers. What is more, we also presented a correlative analysis between serum nitric oxide level and clinical and radiological activity in MS patients. Finally, our aim was also to determine the correlation between nitric oxide concentration and inflammatory parameters in MS patients and healthy controls in the context of the impaired autoimmune system in RRMS subjects.

\section{Material and Methods}

2.1. MS Patients and Healthy Controls. Sixty-three MS patients and 10 healthy subjects were enrolled in a case-control prospective study. All of the study participants were recruited from the Department of Neurology in Zabrze, Medical University of Silesia, Poland. For the present study we chose a group of patients followed up between January and March 2015. All patients had been diagnosed with RRMS, according to the McDonald criteria (2010), and were divided into six study groups:

RRMS INF- $\beta$ la group: 22 patients who received interferon $\beta$ la (Avonex) applied once per week at the dose of $30 \mu \mathrm{g}$ as an intramuscular injection.

RRMS INF- $\beta$ lb group: 17 patients treated with subcutaneous injections of interferon $\beta 1 \mathrm{~b}$ (Extavia or Betaferon) every other day at the dose of $250 \mu \mathrm{g}$.

RRMS-GA group: 5 patients treated with glatiramer acetate (Copaxone) $20 \mathrm{mg}$ subcutaneously daily.

RRMS-NT group: 7 patients who were given NT (Tysabri) intravenously at the dose of $300 \mathrm{mg}$ once per month.

RRMS-FG group: 12 patients were treated with $0.5 \mathrm{mg}$ FG (Gilenya) p.o. daily.

CG (control group): 10 healthy controls age- and sexmatched with the patient group. Healthy controls were diagnosed with insignificant paresthesia in the Department of Neurology and were the residents of the Upper Silesia region and presented lifestyle similar to the study population with MS. None of the patients was treated due to any chronic disease nor did the subjects take any medication permanently.

To reach the goals of our study we divided the subjects into the following two groups: LI group: 44 patients who received the first-line DMT (INF- $\beta 1 \mathrm{a}$, INF- $\beta 1 \mathrm{~b}$, or GA); LII group: 19 patients treated with the second-line DMT (NT or FG).

2.2. Inclusion and Exclusion Criteria. The inclusion criteria were as follows: age 18-55 years, RRMS according to the McDonald criteria (2010), DMT at least 6 months, and signed written informed consent. The exclusion criteria were as follows: different clinical forms of MS other than RRMS, that is, primary progressive, secondary progressive, relapsingprogressive or clinically isolated syndrome, immunosuppressant treatment, active or symptomatic infection or diagnosis of any disease and/or intake of medication which affect inflammatory process, intake of dietary supplements, vitamins, or antioxidative substances 6 months prior to the study and lack of signed informed consent. 
TABle 1: Demographic and clinical characteristics of the study group, depending on different types of disease-modifying therapy.

\begin{tabular}{|c|c|c|c|c|c|c|c|}
\hline Parameters & IFN- $\beta 1 a^{\mathrm{f}}$ & IFN- $\beta 1 \mathrm{~b}$ & $\mathrm{GA}^{\mathrm{g}}$ & $\mathrm{NT}^{\mathrm{h}}$ & $\mathrm{FG}^{\mathrm{i}}$ & $\mathrm{CG}^{\mathrm{j}}$ & $p$ \\
\hline$N$ & 22 & 17 & 5 & 7 & 12 & 10 & \\
\hline Age [years] & $41.3 \pm 11.7$ & $44.8 \pm 10.7$ & $48.6 \pm 12.4$ & $33.9 \pm 7.7$ & $42.3 \pm 13.9$ & - & 0.27 \\
\hline Age at the beginning of $\mathrm{DMT}^{\mathrm{a}}$ [years] & $38.8 \pm 11.1$ & $42.1 \pm 10.8$ & $46.4 \pm 12.8$ & $32.3 \pm 7.9$ & $40.7 \pm 13.5$ & $39.3 \pm 3.5$ & 0.38 \\
\hline Age [\% of females] & 68.2 & 82.4 & 60.0 & 85.7 & 58.3 & 60.0 & 0.62 \\
\hline Time of MS [months] & $57.1 \pm 30.8$ & $85.8 \pm 72.7$ & $90.2 \pm 31.3$ & $81.6 \pm 29.3$ & $85.1 \pm 33.3$ & - & 0.14 \\
\hline Time of DMT [months] & $29.6 \pm 13.7$ & $31.4 \pm 15.1$ & $26.0 \pm 21.8$ & $16.7 \pm 6.5$ & $18.7 \pm 7.6$ & - & 0.15 \\
\hline EDSS $^{\mathrm{b}}[$ score $]$ & $2.5 \pm 1.07$ & $2.41 \pm 0.87$ & $2.60 \pm 1.08$ & $2.79 \pm 1.11$ & $3.08 \pm 1.24$ & - & 0.83 \\
\hline $\operatorname{ARR}^{c}[n]$ & $0.41 \pm 0.59$ & $0.35 \pm 0.61$ & $0.20 \pm 0.45$ & $0.00 \pm \mathrm{ND}$ & $0.50 \pm 0.80$ & - & 0.58 \\
\hline $\mathrm{T} 2 \mathrm{MRI}^{\mathrm{d}}$ lesions (from the time of diagnosis) $[n]$ & $1.14 \pm 0.47$ & $1.18 \pm 0.39$ & $1.00 \pm \mathrm{ND}$ & $1.00 \pm \mathrm{ND}$ & $1.08 \pm 0.29$ & - & 0.76 \\
\hline New MRI lesions (within the last year) $[n]$ & $0.09 \pm 0.29$ & $0.06 \pm 0.24$ & $0.20 \pm 0.45$ & $0.00 \pm \mathrm{ND}$ & $0.33 \pm 0.65$ & - & 0.52 \\
\hline $\mathrm{Gd}+{ }^{\mathrm{e}}$ MRI lesions (from the time of diagnosis) $[n]$ & $0.05 \pm 0.21$ & $0.12 \pm 0.33$ & $20.4 \pm 45.1$ & $0.00 \pm \mathrm{ND}$ & $0.83 \pm 1.75$ & - & 0.20 \\
\hline New Gd+ MRI lesions (within the last year) $[n]$ & $0.05 \pm 0.21$ & $0.06 \pm 0.24$ & $0.2 \pm 0.45$ & $0.00 \pm \mathrm{ND}$ & $0.83 \pm 1.75$ & - & 0.35 \\
\hline
\end{tabular}

a: disease-modifying therapy; b: Expanded Disability Status Scale; c: annual relapse rate; d: magnetic resonance imaging; e: Gadovist; f: interferon beta; g: glatiramer acetate; h: natalizumab; i: fingolimod; j: control group.

2.3. Study Protocol. The diagnosis of MS was based on neurological examination, disease history, and magnetic resonance imaging (MRI) findings using the McDonald criteria (2010). The severity of the clinical course in MS patients ranged from 1.0 to 4.5 and was estimated by the Expanded Disability Status Scale (EDSS).

2.4. MRI Examination. MRI was performed using the $1.5 \mathrm{~T}$ scanner imaging (General Electric HDx USA) and the standard head protocol for MS patients [multiple planes, slice thickness of $5 \mathrm{~mm}$, contrast media: Gd (Gadovist), and additional postcontrast 3DT2 sequences $(1 \mathrm{~mm}$ slice thickness) head MRI]. MRI examination was carried out in all MS patients in compliance with the standard clinical protocol.

2.5. Laboratory Assays. Biochemical assays were measured from fasting serum samples taken in the morning. Blood samples were collected between 7.00 and 8.00 and examined microscopically. To eliminate insoluble substances, the samples were centrifuged 30 minutes after collection, frozen, and stored until laboratory measurements.

2.5.1. Serum IL-1 $\beta / I L-1 F 2$ Concentration. Serum IL-1 $\beta / I L-1 F 2$ concentration was determined according to the Quantikine Human IL-1 $\beta$ immunoassay designed to measure IL- $1 \beta$ in cell culture supernates, serum, and plasma. Solid Phase Sandwich enzyme-linked immunosorbent assay (ELISA) with an assay range of 3.9-250 pg/mL was used in the present study.

2.5.2. NO Assay. The measurement of $\mathrm{NO}$ was performed to estimate $\mathrm{NO}$ metabolites known as nitrite and nitrate $\left(\mathrm{NO}_{x}\right)$, whose concentration was used as a marker of $\mathrm{NO}$ production and iNOS activity. $\mathrm{NO}_{x}$ level was expressed as $\mu \mathrm{M}$. After chemical reduction of nitrate to nitrite using the Griess reaction, we employed the method of Navaro-Gonzalvez et al.

2.5.3. Serum C-Reactive Protein Level. C-reactive protein (CRP), an acute phase protein, was measured with the use of the dry chemistry immunological method and a VITROS 250 analyzer (Ortho Clinical Diagnostics, Johnson and Johnson, USA). Based on the hospital laboratory reference range, CRP concentration above $5 \mathrm{mg} / \mathrm{L}$ was considered abnormal.

2.6. Statistical Analysis. The continuous variables were presented as the means and standard deviations. The categorical variables were shown as percentages. The groups were compared using the nonparametric Mann-Whitney $U$ or the Kruskall-Wallis test for continuous variables and the chi-square test for categorical data. Yates' correction was applied when appropriate. The relationships between variables were tested using the Spearman rank correlation analysis. Statistical significance was defined as $p<0.05$. All statistical analyses were performed using Statistica 12 (Statsoft, Tulsa, Oklahoma, USA).

\section{Results}

Demographic and clinical characteristics of the studied subjects were summarized in Table 1. All patients and the CG were age- and sex-matched $(p>0.05)$. No statistically significant differences were observed between 5 RRMS groups, depending on the type of DMT (INF- $\beta 1 \mathrm{a}, \mathrm{INF}-\beta 1 \mathrm{~b}, \mathrm{GA}, \mathrm{NT}$, and FG) with respect to age, age at the beginning of DMT, sex, MS duration, time of DMT, EDSS, T2 and Gd+ (Gadoliniumenhancing) lesions in MRI at the time of diagnosis, new T2 lesions, and new T1 lesions observed within the previous year prior to the enrollment in the study $(p>0.05)$.

The results of the demographic and clinical characteristics of LI and LII groups were presented in Table 2. We demonstrated a longer duration of MS in LII as compared to the LI group ( $p=0.048$ ). Patients belonging to the LI group had a longer treatment duration of MS as compared to the LII group $(p=0.005)$. No differences in the other above-mentioned parameters were noted. Serum IL-1 $\beta / \mathrm{IL}-1 F 2$ concentration was similar in healthy controls, RRMS IFN- $\beta 1$, RRMS IFN$\beta 1 \mathrm{~b}, \mathrm{RRMS}-\mathrm{GA}, \mathrm{RRMS}-\mathrm{NT}$, and RRMS-FG groups (1.64 \pm $0.47,1.43 \pm 0.34,1.56 \pm 0.60,1.71 \pm 0.41,2.19 \pm 1.27$, and $1.51 \pm 0.56 \mathrm{pg} / \mathrm{mL}, p=0.68$, resp.) as well as in the DMT 
TABLE 2: Demographic and clinical characteristics of the study group, depending on the line of disease-modifying therapy.

\begin{tabular}{|c|c|c|c|c|}
\hline Parameters & LI & LII & $\mathrm{CG}^{\mathrm{j}}$ & $p$ \\
\hline$N$ & 44 & 19 & 10 & - \\
\hline \multirow{2}{*}{$\mathrm{DMT}^{\mathrm{a}}[n]$} & IFla/IF1b ${ }^{\mathrm{f}} / \mathrm{GA}^{\mathrm{g}}$ & $\mathrm{NT}^{\mathrm{h}} / \mathrm{FG}^{\mathrm{i}}$ & \multirow{2}{*}{-} & \multirow{2}{*}{-} \\
\hline & $22 / 17 / 5$ & $7 / 12$ & & \\
\hline Age [years] & $43.5 \pm 11.4$ & $39.2 \pm 12.4$ & - & 0.11 \\
\hline Age at the beginning of DMT [years] & $40.9 \pm 11.2$ & $37.6 \pm 12.2$ & $39.3 \pm 2.47$ & 0.23 \\
\hline Age [\% of females] & 72.7 & 68.4 & 60.0 & 0.72 \\
\hline Time of MS [months] & $72.0 \pm 52.4$ & $87.8 \pm 31.1$ & - & 0.048 \\
\hline Time of DMT [months] & $29.9 \pm 14.9$ & $17.9 \pm 7.1$ & - & 0.005 \\
\hline EDSS $^{\mathrm{b}}$ [score] & $2.48 \pm 0.98$ & $2.97 \pm 1.17$ & - & 0.10 \\
\hline $\operatorname{ARR}^{c}[n]$ & $0.36 \pm 0.57$ & $0.32 \pm 0.67$ & - & 0.51 \\
\hline T2 MRI ${ }^{\mathrm{d}}$ lesions (from the time of diagnosis) $[n]$ & $1.14 \pm 0.41$ & $1.05 \pm 0.23$ & - & 0.45 \\
\hline New MRI lesions (within the last year) $[n]$ & $0.09 \pm 0.29$ & $0.21 \pm 0.54$ & - & 0.42 \\
\hline $\mathrm{Gd}+{ }^{\mathrm{e}}$ MRI lesions (from the time of diagnosis) $[n]$ & $2.39 \pm 15.2$ & $0.53 \pm 1.43$ & - & 0.60 \\
\hline New Gd+ MRI lesions (within the last year) $[n]$ & $0.07 \pm 0.25$ & $0.53 \pm 1.43$ & - & 0.24 \\
\hline
\end{tabular}

A: disease-modifying therapy; b: Expanded Disability Status Scale; c: annual relapse rate; d: magnetic resonance imaging; e: Gadovist; f: interferon beta; g: glatiramer acetate; h: natalizumab; i: fingolimod; j: control group.

TABLE 3: Laboratory assays in the study group, depending on the type of disease-modifying therapy without the assessment of healthy controls.

\begin{tabular}{|c|c|c|c|c|c|c|}
\hline Parameters & IFN- $\beta 1 \mathrm{a}^{\mathrm{d}}$ & IFN- $\beta 1 \mathrm{~b}$ & $\mathrm{GA}^{\mathrm{e}}$ & $\mathrm{NT}^{\mathrm{f}}$ & $\mathrm{FG}^{\mathrm{g}}$ & $p$ \\
\hline$N$ & 22 & 17 & 5 & 7 & 12 & \\
\hline $\mathrm{NO}_{x}{ }^{\mathrm{a}}[\mu \mathrm{M}]$ & $2.25 \pm 0.71$ & $1.90 \pm 0.69$ & $1.58 \pm 0.35$ & $1.65 \pm 0.31$ & $1.52 \pm 0.31$ & 0.018 \\
\hline IL-1 $\beta / \mathrm{IL}-1 \mathrm{~F} 2^{\mathrm{b}}[\mathrm{pg} / \mathrm{mL}]$ & $1.43 \pm 0.34$ & $1.56 \pm 0.60$ & $1.71 \pm 0.41$ & $2.19 \pm 1.27$ & $1.51 \pm 0.56$ & 0.78 \\
\hline $\mathrm{CRP}^{\mathrm{c}}[\mathrm{mg} / \mathrm{L}]$ & $2.43 \pm 3.14$ & $1.88 \pm 1.71$ & $1.72 \pm 1.12$ & $0.95 \pm 0.79$ & $1.81 \pm 2.5$ & 0.79 \\
\hline
\end{tabular}

A: nitric oxide (nitrite and nitrate); b: interleukin; c: C-reactive protein; d: interferon beta; e: glatiramer acetate; f: natalizumab; g: fingolimod.

groups without CG evaluation (Table 3). No differences were found in the serum level of IL-1 $\beta / \mathrm{IL}-1 \mathrm{~F} 2$ between LI and LII groups and healthy controls $(1.51 \pm 0.46,1.76 \pm 0.92$, and $1.64 \pm 0.47 \mathrm{pg} / \mathrm{mL}, p=0.47$, resp.) as well as between LI and LII without CG assessment (Table 4). However, in LI serum concentration of $\mathrm{NO}_{x}$ was significantly higher compared LII (Table 4). The same results were noted in the analysis between LI, LII, and CG $(2.04 \pm 0.70,1.57 \pm 0.31$, and $1.50 \pm 0.16 \mu \mathrm{M}, p=$ 0.009 , resp.). There were also differences in the level of $\mathrm{NO}_{x}$ in healthy controls, RRMS IFN- $\beta 1 \mathrm{a}, \mathrm{RRMS}$ IFN- $\beta 1 \mathrm{~b}, \mathrm{RRMS}-$ GA, RRMS-NT, and RRMS-FG groups $(1.50 \pm 0.16,2.25 \pm 0.71$, $1.90 \pm 0.69,1.58 \pm 0.35,1.65 \pm 0.31$, and $1.52 \pm 0.31 \mu \mathrm{M}$, $p=0.003$, resp.) as well as in the DMT groups without CG evaluation (Table 3). Serum CRP level was similar in the CG, INF- $\beta 1 \mathrm{a}, \mathrm{INF}-\beta 1 \mathrm{~b}, \mathrm{GA}, \mathrm{NT}$, and FG groups $(1.19 \pm 0.55$, $2.43 \pm 3.14,1.88 \pm 1.71,1.72 \pm 1.12,0.95 \pm 0.79$, and $1.81 \pm$ $2.5 \mathrm{mg} / \mathrm{L}, p=0.78$, resp.) and in the DMT groups without CG evaluation (Table 3). No differences in CRP concentration between LI, LII, and the CG were observed $(2.13 \pm 2.47$, $1.49 \pm 2.05$, and $1.19 \pm 0.55, p=0.52$, resp.). However, excluding the CG, a comparison between LI and LII revealed a trend to a higher CRP level in LI (Table 4). Considering the EDSS, we estimated serum concentration of $\mathrm{NO}_{x}$, IL$1 \beta / \mathrm{IL}-1 \mathrm{~F} 2$, and CRP in two groups, depending on the EDSS results $(0-3$ and $>3)$. No significant differences were found in this analysis (Table 5). Furthermore, we did not observe the correlation between $\mathrm{NO}_{x}$ and the EDSS, annual relapse rate, and the number of MRI lesions measured in the whole
MS population, in LI and LII groups (Table 6). However, a significant correlation between serum $\mathrm{NO}_{x}$ level and MS duration was observed, which was confirmed in the whole study population $(R=-0.3046, p<0.05)$ (Figure 1) and in the LI group $(R=-0.36, p<0.05)$ (Figure $2(\mathrm{a}))$, unlike in the LII group $(R=0.24, p>0.05)$ (Figure $2(b))$. We also found a higher serum level of $\mathrm{NO}_{x}$ when the duration of RRMS was shorter than the median time ( $<59.5$ months) as compared to the disease duration of $>59.5$ months $(2.11 \pm 0.73$ and $1.67 \pm 0.45, p=0.01$, resp.). Measurements of IL- $1 \beta /$ IL$1 \mathrm{~F} 2$ did not show differences in the groups formed, depending on MS duration $(1.53 \pm 0.61$ versus $1.63 \pm 0.67, p=0.65$ for $<59.5$ months and $>59.5$ months, resp.). Furthermore, there were no differences in CRP concentration estimated in the groups formed, depending on MS duration (2.14 \pm 2.91 versus $1.75 \pm 1.67, p=0.73$ for $<59.5$ months and $>59.5$ months, resp.). Interestingly, we showed a correlation between serum CRP and IL-1 $\beta /$ IL-1F2 concentration in healthy controls ( $r=$ 0.65091, $p<0.05$ ), whereas no correlations were found in the whole MS population $(R=-0.1102, p>0.05)$ (Figure 3$)$ in LI (Figure 4(a)) and in LII (Figure 4(b)).

\section{Discussion}

The results of our study demonstrate that MS patients treated with IFN- $\beta 1 \mathrm{a}$ - IFN- $\beta$ 1b had significantly higher serum $\mathrm{NO}_{x}$ levels as compared to the healthy controls. Similarly, previous reports discussed the measurement of $\mathrm{NO}_{x}$ levels $[19,37]$. 
TABLE 4: Laboratory assays in the study group, depending on the line of disease-modifying therapy.

\begin{tabular}{lccc}
\hline & LI & LII & $p$ \\
& $N=44$ & $N=19$ & 0.003 \\
$\mathrm{NO}_{x}{ }^{\mathrm{a}}[\mu \mathrm{M}]$ & $2.04 \pm 0.70$ & $1.57 \pm 0.31$ & 0.81 \\
$\mathrm{IL}-1 \beta / \mathrm{IL}-1 \mathrm{~F} 2^{\mathrm{b}}[\mathrm{pg} / \mathrm{mL}]$ & $1.51 \pm 0.46$ & $1.76 \pm 0.92$ & 0.06 \\
$\mathrm{CRP}^{\mathrm{c}}[\mathrm{mg} / \mathrm{L}]$ & $2.13 \pm 2.47$ & $1.49 \pm 2.05$ & 0.06 \\
\hline
\end{tabular}

A: nitric oxide (nitrite and nitrate); b: interleukin; c: C-reactive protein.

TABLE 5: Laboratory assays in lower and higher Expanded Disability Status Scale groups.

\begin{tabular}{lccr}
\hline & EDSS $^{\mathrm{a}} 0-3$ [score] & EDSS $>3$ [score] & N \\
& $N=46$ & $1.70 \pm 0.53$ & \\
\hline $\mathrm{NO}_{x}{ }^{\mathrm{b}}[\mu \mathrm{M}]$ & $1.97 \pm 0.67$ & $1.78 \pm 0.84$ & 0.10 \\
$\mathrm{IL}-1 \beta / \mathrm{IL}-1 \mathrm{~F} 2^{\mathrm{c}}[\mathrm{pg} / \mathrm{mL}]$ & $1.51 \pm 0.54$ & $1.79 \pm 2.12$ & 0.32 \\
$\mathrm{CRP}^{\mathrm{d}}[\mathrm{mg} / \mathrm{L}]$ & $2.0 \pm 2.45$ & 0.76 \\
\hline
\end{tabular}

A: Expanded Disability Status Scale; b: nitric oxide (nitrite and nitrate); c: interleukin; d: C-reactive protein.

Furthermore, other studies failed to observe a higher $\mathrm{NO}_{x}$ concentration in the serum of MS patients as compared to healthy controls $[18,21,38]$. Different outcomes were probably related to the various neurological and clinical status of MS patients, another form of the disease, different lifestyle, diet, or pharmacological interactions.

However, $\mathrm{NO}_{x}$ is considered a major factor in the pathophysiology of MS [21]. It is produced as a result of a catalytic conversion of L-arginine to citrulline by endothelial and neuronal iNOS. Concomitantly, arginase involved in Larginine conversion leads to the production of ornithine, favoring cellular regeneration [39]. Additionally, $\mathrm{NO}_{x}$ creates a family of toxic molecules known as RNS. Finally, it leads to nitrosylation resulting in cellular death [3]. High levels of $\mathrm{NO}_{x}$ are implicated in active MS lesions produced by astrocytes and microglia, as an after-effect of the expression of iNOS. It is believed that $\mathrm{NO}_{x}$ causes tissue damage and blocks axonal conduction and results in degeneration [17]. On the other hand, under normal conditions $\mathrm{NO}$ is known to be a signaling molecule regulating cellular functions, neurotransmission, vasodilatation, and immune response, mediated by cyclic guanosine monophosphate. In contrast, toxic properties of $\mathrm{NO}_{x}$ are related to the above-mentioned RNS. Therefore, it is suggested that NO plays a conflicting role in the pathophysiology of neuroimmunological diseases such as MS with pro-and anti-inflammatory activities, depending on local concentrations. Under physiological conditions high concentration of $\mathrm{NO}$ is considered to be a neuroprotective factor [39]. However, MS is a chronic inflammatory disorder with impaired immune regulation, resulting in a high activity of serum $\mathrm{NO}_{x}$ in MS patients. It has recently been reported that persistent inflammatory response results in greater iNOS expression and NO production, while arginase activity predominates under physiological conditions with increased Th2 cytokine expression [40]. It seems that the data from our study are consistent with these findings.

Despite the involvement of various inflammatory mediators in immunity, only a few cytokines seem to be essential for the development of CNS autoimmunity. Recently IL1 has been recognized as a pivotal factor in experimental

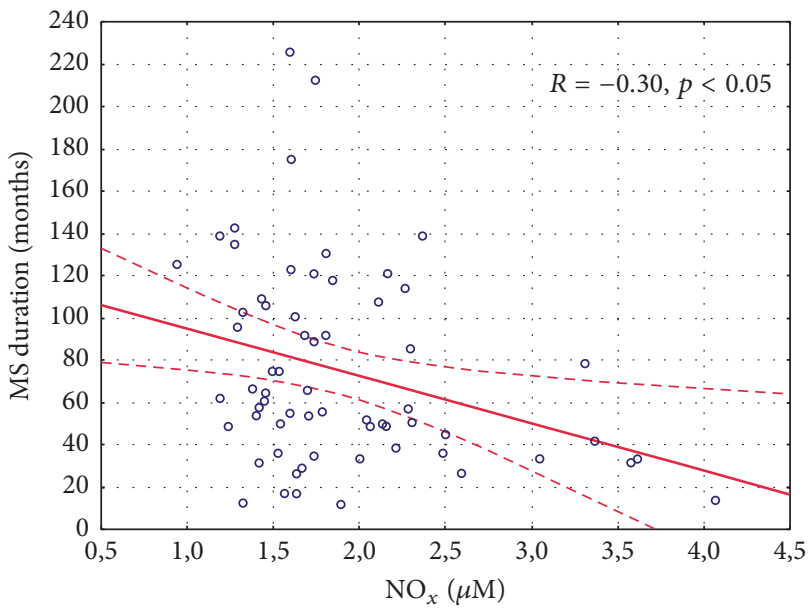

FIgURE 1: Correlation between nitric oxide (nitrite and nitrate) plasma concentration and the time of multiple sclerosis in the whole study population.

autoimmune encephalomyelitis and MS, initiating and expanding the disease [41]. There is a growing evidence for the impact of IL-1 on neuroinflammation [42]. Additionally, IL-1 induced Bhlhe40 expression in polarized Th17 cells in a model of autoimmune neuroinflammation [43]. Based on the above findings, IL-1 $\beta / \mathrm{IL}-1 \mathrm{~F} 2$ is a proinflammatory cytokine which we initially considered a potential factor activating inflammatory process in MS patients.

In the CNS of MS patients continued inflammatory process with impaired immune regulation is observed. This phenomenon results in high activity of serum $\mathrm{NO}_{x}$. Concomitantly, it has recently been reported that persistent inflammatory response results in greater iNOS expression [40]. Reports confirm the damage to the inflamed nerve tissue which is due to $\mathrm{NO}_{x}$. Impaired blood-brain barrier is the result of vasodilatation, which promotes the passage of inflammatory cells to the CNS [44, 45]. Consequently, increased permeability of the blood-brain barrier at the sites of inflammatory lesions is observed [46]. On the other hand, 
TABLE 6: Correlation between nitric oxide (nitrate and nitrite) serum levels and clinical and radiological parameters measured in all multiple sclerosis patients and in groups under first and second line of disease-modifying therapies.

\begin{tabular}{|c|c|c|c|}
\hline $\mathrm{NO}_{x}{ }^{\mathrm{a}}[\mu \mathrm{M}]$ & $M S^{f}$ patients & LI & LII \\
\hline$N$ & 63 & 44 & 19 \\
\hline EDSS $^{\mathrm{b}}$ [points] & -0.19 & -0.10 & -0.29 \\
\hline $\operatorname{ARR}^{c}[n]$ & -0.007 & -0.12 & -0.19 \\
\hline T2 MRI $^{\mathrm{d}}$ lesions (from the time of diagnosis) $[n]$ & -0.05 & 0.11 & -0.28 \\
\hline New MRI lesions (within the last year) $[n]$ & -0.05 & 0.13 & -0.41 \\
\hline $\mathrm{Gd}+{ }^{\mathrm{e}} \mathrm{MRI}$ lesions (from the time of diagnosis) $[n]$ & -0.14 & -0.03 & -0.42 \\
\hline New Gd+ MRI lesions (within the last year) $[n]$ & -0.22 & 0.19 & -0.42 \\
\hline
\end{tabular}

${ }^{*} p>0.05$ in all analysis.

A: nitric oxide (nitrite and nitrate); b: Expanded Disability Status Scale. C: annual relapse rate; d: magnetic resonance imaging; e: Gadovist; f: multiple sclerosis.

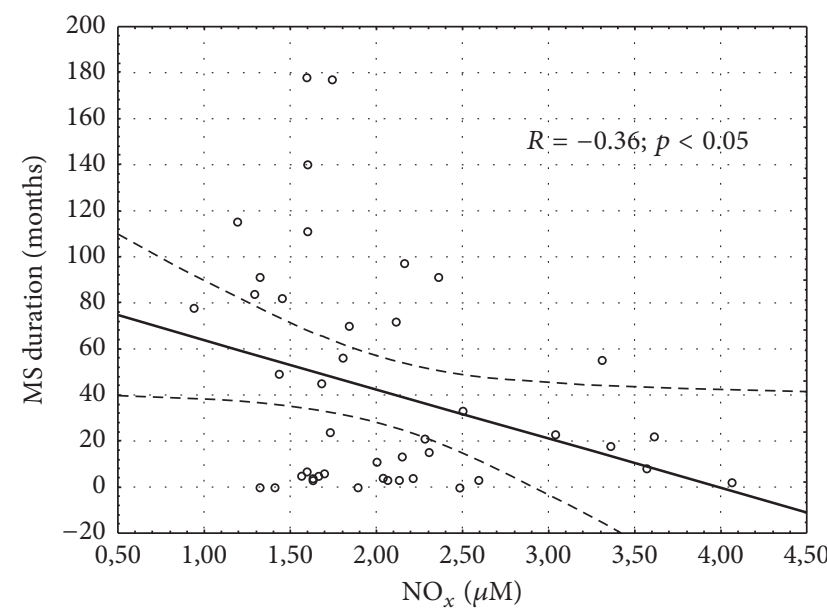

(a)

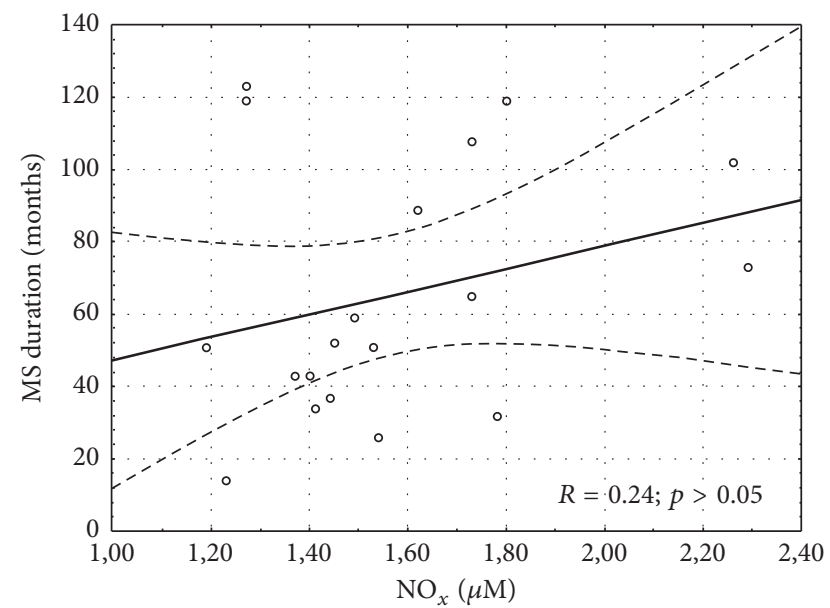

(b)

FIGURE 2: Correlation between nitric oxide (nitrite and nitrate) plasma concentration and the time of multiple sclerosis in the patients who received the first- (a) and second-line disease-modifying therapy (b).

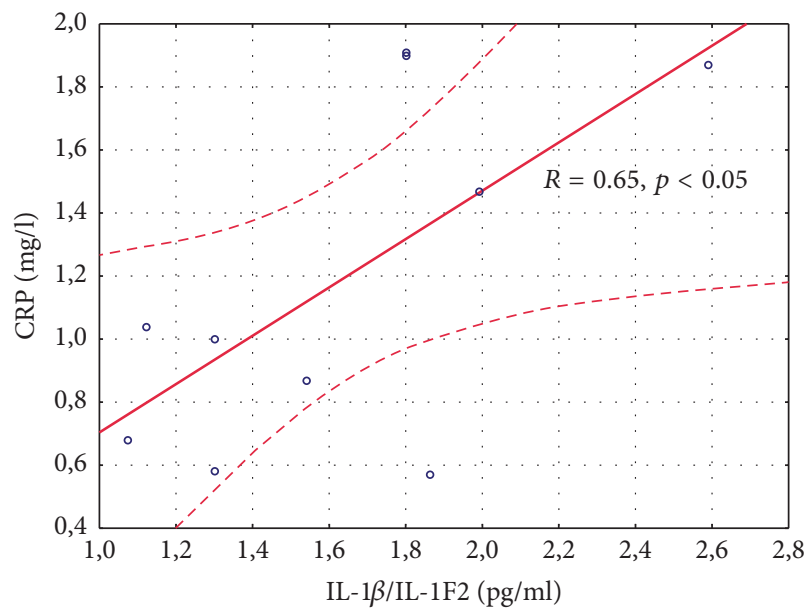

(a)

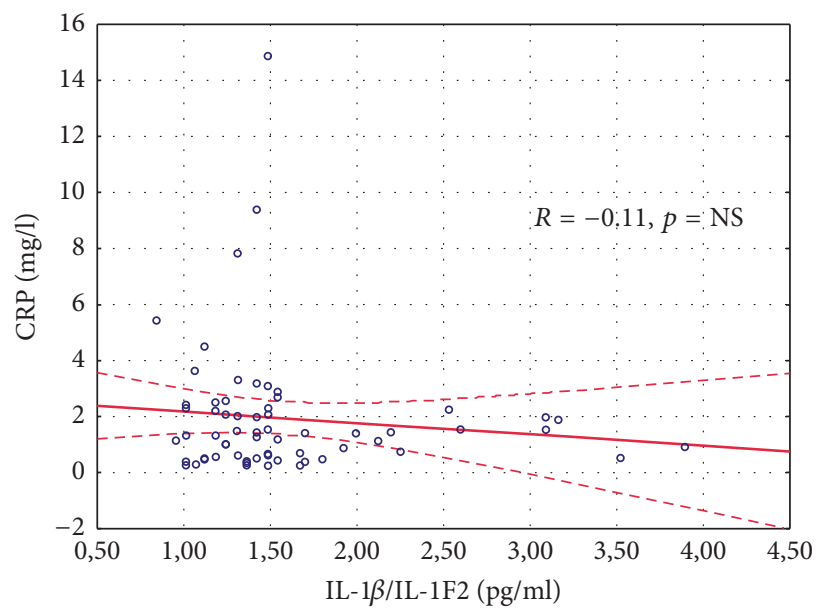

(b)

FIGURE 3: Correlation between serum C-reactive protein and IL-1 $\beta / \mathrm{IL}-1 \mathrm{~F} 2$ concentration in healthy controls (a) and in the whole study multiple sclerosis population (b). 


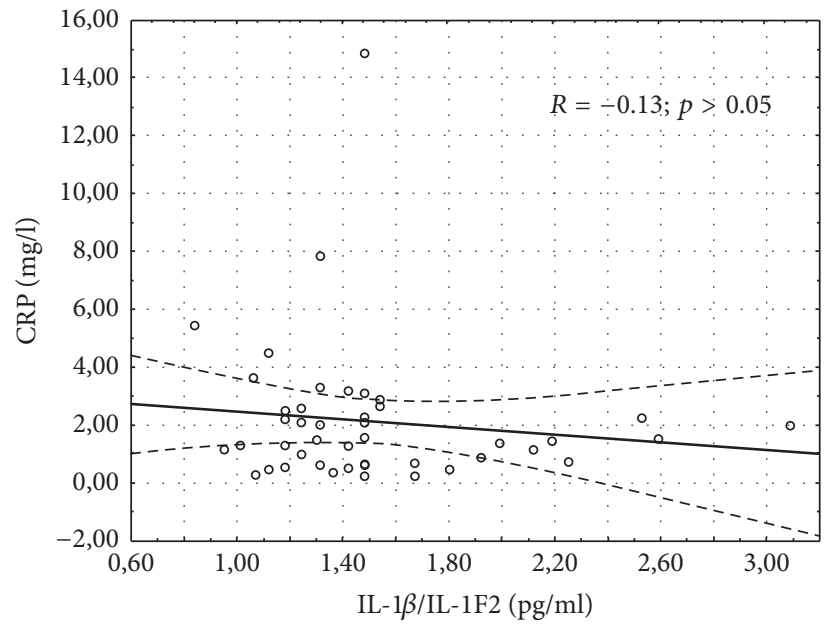

(a)

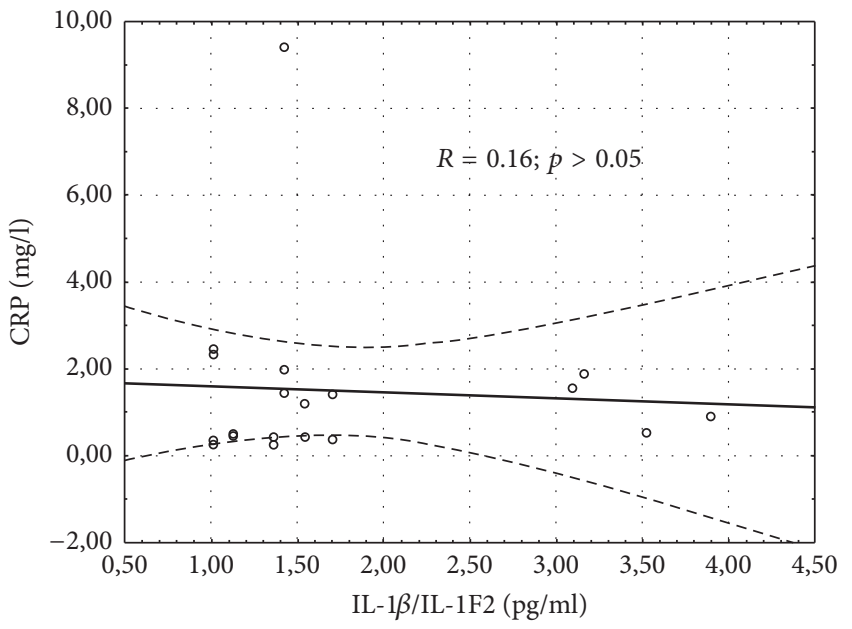

(b)

FIGURE 4: Correlation between serum C-reactive protein and IL-1 $\beta / I L-1 F 2$ concentration in the patients who received the first- (a) and secondline disease-modifying therapy (b).

it is well documented that DMTs (INF- $\beta 1 \mathrm{a}$, INF- $\beta 1 \mathrm{~b}$, and GA) slow the progressive nerve damage thus changing the course of the disease. This mechanism is still poorly understood. However, DMTs are suspected to downregulate inflammatory cytokines and reduce blood-brain barrier permeability and T-cell migration into the CNS. The second-line agents (e.g., NT monoclonal antibody) decrease inflammatory response by blocking receptors on white blood cells which allow them to enter the brain and the spinal cord [3]. Fingolimod is able to interfere with the inflammatory phase of MS through the prevention of lymphocyte infiltration into the CNS [34]. Additionally, FG affects positively damaged myelin regions binding to S1P receptor on oligodendrocytes [47].

In our study we assumed that higher $\mathrm{NO}_{x}$ level is linked to the presence of greater inflammation and nitrosative stress in the serum of MS patients. Therefore, anti-inflammatory effect of DMT resulted in decreased $\mathrm{NO}_{x}$ serum concentration. Considering higher $\mathrm{NO}_{x}$ serum activity obtained from patients treated with the first-line DMT (IFN- $\beta 1 \mathrm{a}$; IFN- $\beta 1 \mathrm{~b}$ ) as compared to the second-line, we could hypothesize that the second-line agents produce a better anti-inflammatory and antinitrosative effect. Additionally, these agents decrease the extent of nerve damage due to $\mathrm{NO}_{x}$ in the inflammatory process. Moreover, based on the analysis of patients under second-line DMT who previously were given the first-line agents, we observed a cumulative anti-inflammatory effect of the first- and second-line DMT in the LII group (serum $\mathrm{NO}_{x}$ was higher compared to the LI group despite the fact that the time of DMT was shorter in LII). On the other hand, there is evidence that MS patients treated with INF$\beta 1 \mathrm{~b}$ had significantly lower concentrations of NO metabolites in the serum as compared to the untreated MS patients [48]. Additionally, a three-year INF- $\beta 1 \mathrm{a}$ or INF- $\beta$ 1b monotherapy is reported to have reduced serum $\mathrm{NO}_{x}$ levels by $77 \%$ and $71 \%$, respectively [49].

Interestingly, we also confirmed that a longer MS duration was correlated with a lower $\mathrm{NO}_{x}$ serum level. At the same time $\mathrm{NO}_{x}$ serum concentration was lower when disease duration was above 59.5 months. It suggests that patients of longer disease duration used more DMT starting from the time of diagnosis in comparison with the subjects of shorter disease duration. On the other hand, decreased $\mathrm{NO}_{x}$ serum activity in patients with disease duration above 59.5 months seems to be justified due to DMT, which limited inflammatory response. However, Ljubisavljevic et al. showed higher serum NO levels of clinical isolated syndrome as compared to the RRMS group, indicating a possible modulation of inflammation during the course of the disease [39]. Nonetheless, RRMS patients had not received interferon within 6 months prior to the study enrollment.

The data from our study showed no correlation between serum $\mathrm{NO}_{x}$ level and the EDSS in MS patients. However, Rejdak et al. reported a correlation between NO metabolites and the EDSS [50]. Similar data showed a positive correlation between $\mathrm{NO}_{x}$ and the EDSS in RRMS patients [51]. Contrary to our study, Rejdak et al. assayed the concentration of $\mathrm{NO}_{x}$ in the cerebrospinal fluid. We did not confirm the correlation between $\mathrm{NO}_{x}$ serum level and new MRI lesions or lesions detected at the time of diagnosis. However, other authors observed that the concentration of $\mathrm{NO}_{x}$ in the cerebrospinal fluid correlated with the volume of $\mathrm{Gd}+$ lesions on MRI [52]. It probably corresponds better with axonal degeneration, the EDSS, and MRI lesions as compared to $\mathrm{NO}_{x}$ serum activity.

Multiple sclerosis is defined as a chronic inflammation of the CNS, while active inflammation and the breakdown of the blood-brain barrier may be detected in MRI brain lesions in MS patients [53-55]. Inflammation in MS is a dynamic process presented at all stages of the disease [56] and the course of MS depends on the location within the brain (perivascular, meningeal, and parenchymal) and on the clinical aspects such as age or disease duration [57]. Consequently, the evaluation of inflammation remains difficult. Furthermore, inflammatory biomarkers assayed in our study (IL-1 $\beta /$ IL1F2 and CRP) did not differ in all the groups divided, depending on the types of DMT and in the CG. Previously, Soilu-Hänninen et al. also reported that CRP values in MS 
patients and in healthy controls were similar [58], whereas the results of another study showed significantly increased levels of CRP in RRMS subjects without DMT and in treated RRMS patients [24]. In our study we indicated a trend ( $p=$ $0.06)$ toward a higher CRP concentration in the group treated with the first-line DMT as compared to the patients using second-line agents. As in the case of $\mathrm{NO}_{x}$ level, we should hypothesize better reduction in inflammation using the second-line DMT. Finally, we proved a positive correlation between CRP and IL-1 $\beta / \mathrm{IL}-1 \mathrm{~F} 2$ serum concentration as the inflammatory indicators in healthy controls. This correlation was not confirmed in the whole MS population. It seems that this phenomenon was due to the immune dysregulation and subsequent impaired inflammatory response in MS patients. As a result, dendritic cells have an activated phenotype in subjects with MS and migrate across the blood-brain barrier inducing differentiation of T cells into proinflammatory Th1 and Th17 lymphocytes. Moreover, activated macrophages and microglia produce other proinflammatory cytokines and nitric and oxide radicals which being responsible for the failure of the CNS in MS patients [32].

Using antioxidant therapy may be beneficial in addition to DMTs. There are already reports on the antioxidant protection of biological targets against ROS. Hence, it has been suggested that antioxidants may be attractive potential therapeutic agents to prevent neuronal damage caused by ROS [59]. However, there is some evidence indicating complex interactions between exogenous and endogenous antioxidant mechanisms. Under physiological conditions, this balance protects from nitrosative and oxidative stress. On the other hand, it is well known that nuclear factor erythroid-derived 2-like 2 (Nrf2) participates in the antioxidant defense. This molecule is strongly involved in regulation of many different genes linked to the inflammatory processes, stem cell regulation, or regeneration [60]. Activation of $\mathrm{Nrf} 2$ was reported to inhibit production and secretion of inflammatory cytokines [61]. What is more, Nrf2 activity depends on the presence of radicals. In experimental autoimmune encephalomyelitis (EAE) low levels of antioxidants ( $\gamma$-glutamylcysteine ligase and glutathione synthetase) were observed, which correlated with a diminished expression of Nrf2 [62]. The exogenous antioxidant treatment could potentially reduce nitrosative and oxidative stress. There are some reports on the increased induction of Nrf2-antioxidant mediated genes after vitamin E supplementation [63]. However, it was also observed that anti-inflammatory and antioxidant activities of tocopherols are independent of Nrf2 in mice [64]. Such different results found in the literature may question beneficial effects and clinical efficacy of exogenous antioxidants [65]. Adding antioxidant therapy to the DMTs (with their anti-inflammatory properties) should be well thought out and balanced. Certainly, it requires further investigation in clinical trials.

\section{Limitation of the Study}

Although the present study has yielded some preliminary findings, its design is not without flaws. We had to interpret the results very carefully due to the small sample size. We included a relatively small group of MS patients divided into much smaller subgroups depending on DMTs. Despite demonstrating the correlation between $\mathrm{NO}_{x}$ levels and MS duration for the whole MS study population and LI, such a correlation for LII could not be confirmed. We suppose that this result was due to the LII group which included significantly fewer patients compared to the LI group (19 subjects versus 44 , resp.). Hence, to finally confirm $\mathrm{NO}_{x}$ serum level as a biomarker of MS duration further investigation is required. However, the small sample size was related to the restrictive inclusion criteria used to carefully select MS patients. Therefore, we are planning to include more participants (patients as well as healthy controls) in the future. Relapsing-remitting MS patients will be divided (as previously) into 5 groups, each including 50, 50, 45, 40, and 40 patients who will receive INF- $\beta 1 \mathrm{a}$, INF- $\beta 1 \mathrm{~b}$, glatiramer acetate, natalizumab, and fingolimod, respectively. Although we described altered serum $\mathrm{NO}_{x}$ level expression in MS patients it would be beneficial to evaluate some other nitrosative stress biomarkers. Certainly, we would like to measure other biomarkers of RNS production, such as 4,5-diaminofluorescein (DAF-2), 2,3-diaminonaphthalene (DAN), 2,3-naphthotriazole (NAT), nitrosothiols, or nitric oxide synthase (NOS) in the serum of MS patients. Consequently, more biomarkers may reflect the RNS production more effectively. To include more subjects in the study group as well as to extend the laboratory assessment we are in the process of recruiting patients from the Department of Neurology in Zabrze, Medical University of Silesia, Poland. Moreover, the assessment of both nitrogen species and inflammatory parameters in the cerebrospinal fluid may be beneficial for further studies.

\section{Conclusions}

We observed higher $\mathrm{NO}_{x}$ activity in MS subjects treated with IFN- $\beta 1 \mathrm{a}$; IFN- $\beta 1 \mathrm{~b}$ as compared to the healthy controls. The results of our study suggest that $\mathrm{NO}_{x}$ serum level may be a biomarker of MS duration. However, our study did not demonstrate disease activity measured by the EDSS, annual relapse rate, and MRI lesions. Considering the hypothesis that serum $\mathrm{NO}_{x}, \mathrm{CRP}$, and IL-1 $\beta / \mathrm{IL}-1 \mathrm{~F} 2$ concentrations could reveal potential efficacy of DMT, we reported only significant differences in $\mathrm{NO}_{x}$ levels depending on various types of DMT. Based on it, we can suggest better reduction of inflammation using the second-line agents of DMT. This issue of MS treatment is intriguing and should be further investigated. Adding antioxidant therapy to the DMTs (with their antioxidative properties) should be well thought out and balanced. Certainly, it requires further investigation in clinical trials.

\section{Ethical Approval}

The study protocol was approved by the local Ethics Committee of the Medical University of Silesia (KNW/0022/KBI/ 130/12). All procedures performed in studies involving human participants were in accordance with the ethical standards of the institutional and/or national research committee and with the 1964 Helsinki declaration and its later amendments or comparable ethical standards. 


\section{Consent}

The experiment was conducted with the human subjects' understanding. Informed consent was obtained from all individual participants in the study.

\section{Competing Interests}

The authors declare that there is no conflict of interests regarding the publication of this paper.

\section{References}

[1] S. V. Ramagopalan and A. D. Sadovnick, "Epidemiology of Multiple sclerosis," Neurologic Clinics, vol. 29, no. 2, pp. 207-217, 2011.

[2] J. Nakahara, M. Maeda, S. Aiso, and N. Suzuki, "Current concepts in multiple sclerosis: autoimmunity versus oligodendrogliopathy," Clinical Reviews in Allergy and Immunology, vol. 42, no. 1, pp. 26-34, 2012.

[3] A. Suneetha and K. Raja Rajeswari, "Role of dimethyl fumarate in oxidative stress of multiple sclerosis: a review," Journal of Chromatography B, vol. 1019, pp. 15-20, 2016.

[4] D. A. Hafler, A. Compston, S. Sawcer et al., "Risk alleles for multiple sclerosis identified by a genomewide study," New England Journal of Medicine, vol. 357, no. 9, pp. 851-862, 2007.

[5] J. L. Huynh and P. Casaccia, "Epigenetic mechanisms in multiple sclerosis: implications for pathogenesis and treatment," The Lancet Neurology, vol. 12, no. 2, pp. 195-206, 2013.

[6] H. Lassmann, "The pathology of multiple sclerosis and its evolution," Philosophical Transactions of the Royal Society B: Biological Sciences, vol. 354, no. 1390, pp. 1635-1640, 1999.

[7] H. Lassmann, "Pathology and disease mechanisms in different stages of multiple sclerosis," Journal of the Neurological Sciences, vol. 333, no. 1-2, pp. 1-4, 2013.

[8] A. P. Kallaur, E. M. V. Reiche, S. R. Oliveira et al., "Genetic, immune-inflammatory, and oxidative stress biomarkers as predictors for disability and disease progression in multiple sclerosis," Molecular Neurobiology, pp. 1-14, 2016.

[9] C. Lucchinetti, W. Brück, J. Parisi, B. Scheithauer, M. Rodriguez, and H. Lassmann, "Heterogeneity of multiple sclerosis lesions: implications for the pathogenesis of demyelination," Annals of Neurology, vol. 47, no. 6, pp. 707-717, 2000.

[10] G. R. Campbell and D. J. Mahad, "Clonal expansion of mitochondrial DNA deletions and the progression of multiple sclerosis," CNS \& Neurological Disorders_Drug Targets, vol. 11, no. 5, pp. 589-597, 2012.

[11] D. Centonze, L. Muzio, S. Rossi, R. Furlan, G. Bernardi, and G. Martino, "The link between inflammation, synaptic transmission and neurodegeneration in multiple sclerosis," Cell Death and Differentiation, vol. 17, no. 7, pp. 1083-1091, 2010.

[12] I. Tasset, E. Agüera, F. Sánchez-López et al., "Peripheral oxidative stress in relapsing-remitting multiple sclerosis," Clinical Biochemistry, vol. 45, no. 6, pp. 440-444, 2012.

[13] S. R. Oliveira, A. P. Kallaur, A. N. C. Simão et al., "Oxidative stress in multiple sclerosis patients in clinical remission: association with the expanded disability status scale," Journal of the Neurological Sciences, vol. 321, no. 1-2, pp. 49-53, 2012.

[14] Y. Song, G. J. Pinniger, A. J. Bakker et al., "Lipopolysaccharideinduced weakness in the preterm diaphragm is associated with mitochondrial electron transport chain dysfunction and oxidative stress," PLoS ONE, vol. 8, no. 9, Article ID 0073457, 2013.

[15] E. Miller, M. Mrowicka, J. Saluk-Juszczak, and M. Ireneusz, "The level of isoprostanes as a non-invasive marker for in vivo lipid peroxidation in secondary progressive multiple sclerosis," Neurochemical Research, vol. 36, no. 6, pp. 1012-1016, 2011.

[16] V. Calabrese, T. E. Bates, and A. M. G. Stella, "NO synthase and NO-dependent signal pathways in brain aging and neurodegenerative disorders: the role of oxidant/antioxidant balance," Neurochemical Research, vol. 25, no. 9-10, pp. 1315-1341, 2000.

[17] M. Koch, J. Mostert, A. Arutjunyan et al., "Peripheral blood leukocyte NO production and oxidative stress in multiple sclerosis," Multiple Sclerosis, vol. 14, no. 2, pp. 159-165, 2008.

[18] S. Ibragic, E. Sofic, E. Suljic, N. Avdagic, A. Bajraktarevic, and I. Tahirovic, "Serum nitric oxide concentrations in patients with multiple sclerosis and patients with epilepsy," Journal of Neural Transmission, vol. 119, no. 1, pp. 7-11, 2012.

[19] M. Roghani, F. Mahboudi, M. A. Saharian et al., "Concentrations of nitric oxide metabolites in the serum of Iranian multiple sclerosis patients," Journal of the Neurological Sciences, vol. 294, no. 1-2, pp. 92-94, 2010.

[20] K. J. Smith and H. Lassmann, "The role of nitric oxide in multiple sclerosis," Lancet Neurology, vol. 1, no. 4, pp. 232-241, 2002.

[21] A. Acar, M. Ugur Cevik, O. Evliyaoglu et al., "Evaluation of serum oxidant/antioxidant balance in multiple sclerosis," Acta Neurologica Belgica, vol. 112, no. 3, pp. 275-280, 2012.

[22] M. Ghabaee, B. Jabedari, N. Al-E-Eshagh, M. Ghaffarpour, and F. Asadi, "Serum and cerebrospinal fluid antioxidant activity and lipid peroxidation in guillain-barre syndrome and multiple sclerosis patients," International Journal of Neuroscience, vol. 120, no. 4, pp. 301-304, 2010.

[23] M. Adamczyk-Sowa, K. Pierzchala, P. Sowa et al., "Melatonin acts as antioxidant and improves sleep in MS patients," Neurochemical Research, vol. 39, no. 8, pp. 1585-1593, 2014.

[24] I. Sadowska-Bartosz, M. Adamczyk-Sowa, S. Galiniak, S. Mucha, K. Pierzchala, and G. Bartosz, "Oxidative modification of serum proteins in multiple sclerosis," Neurochemistry International, vol. 63, no. 5, pp. 507-516, 2013.

[25] I. Sadowska-Bartosz, M. Adamczyk-Sowa, A. Gajewska, and G. Bartosz, "Oxidative modification of blood serum proteins in multiple sclerosis after interferon or mitoxantrone treatment," Journal of Neuroimmunology, vol. 266, no. 1-2, pp. 67-74, 2014.

[26] G. G. Ortiz, M. Á. Macías-Islas, F. P. Pacheco-Moisés et al., "Oxidative stress is increased in serum from Mexican patients with relapsing-remitting multiple sclerosis," Disease Markers, vol. 26, no. 1, pp. 35-39, 2009.

[27] K. J. Smith, "Newly lesioned tissue in multiple sclerosis-a role for oxidative damage?” Brain, vol. 134, no. 7, pp. 1877-1881, 2011.

[28] T. Poyraz, E. Idiman, S. Uysal et al., "The cooling effect on proinflammatory cytokines interferon- $\gamma$, tumor necrosis factor$\alpha$, and nitric oxide in patients with multiple sclerosis," ISRN Neurology, vol. 2013, pp. 1-6, 2013.

[29] S. Moncada and A. Higgs, "The L-arginine-nitric oxide pathway," New England Journal of Medicine, vol. 329, no. 27, pp. 2002-2012, 1993.

[30] S. Ljubisavljevic, I. Stojanovic, S. Vojinovic et al., “The patients with clinically isolated syndrome and relapsing remitting multiple sclerosis show different levels of advanced protein oxidation products and total thiol content in plasma and CSF," Neurochemistry International, vol. 62, no. 7, pp. 988-997, 2013. 
[31] R. Weissert, "The immune pathogenesis of multiple sclerosis," Journal of Neuroimmune Pharmacology, vol. 8, no. 4, pp. 857866, 2013.

[32] N. Grigoriadis and V. van Pesch, "A basic overview of multiple sclerosis immunopathology," European Journal of Neurology, vol. 22, supplement 2, pp. 3-13, 2015.

[33] P. Kivisäkk, B. C. Healy, V. Viglietta et al., "Natalizumab treatment is associated with peripheral sequestration of proinflammatory T cells," Neurology, vol. 72, no. 22, pp. 1922-1930, 2009.

[34] J. Chun and H.-P. Hartung, "Mechanism of action of oral fingolimod (FTY720) in multiple sclerosis," Clinical Neuropharmacology, vol. 33, no. 2, pp. 91-101, 2010.

[35] A. Groves, Y. Kihara, and J. Chun, "Fingolimod: direct CNS effects of sphingosine 1-phosphate (S1P) receptor modulation and implications in multiple sclerosis therapy," Journal of the Neurological Sciences, vol. 328, no. 1-2, pp. 9-18, 2013.

[36] W. M. Carroll, "Clinical trials of multiple sclerosis therapies: improvements to demonstrate long-term patient benefit," Multiple Sclerosis, vol. 15, no. 8, pp. 951-958, 2009.

[37] G. Acar, F. Idiman, E. Idiman, G. Kirkali, H. Çakmakçi, and S. Özakbaş, "Nitric oxide as an activity marker in multiple sclerosis," Journal of Neurology, vol. 250, no. 5, pp. 588-592, 2003.

[38] F. de Bustos, J. A. Navarro, C. de Andrés et al., "Cerebrospinal fluid nitrate levels in patients with multiple sclerosis," European Neurology, vol. 41, no. 1, pp. 44-47, 1999.

[39] S. Ljubisavljevic, I. Stojanovic, R. Pavlovic, and D. Pavlovic, "The importance of nitric oxide and arginase in the pathogenesis of acute neuroinflammation: are those contra players with the same direction?" Neurotoxicity Research, vol. 26, no. 4, pp. 392399, 2014

[40] M. Ahn, W. Yang, H. Kim, J.-K. Jin, C. Moon, and T. Shin, "Immunohistochemical study of arginase-1 in the spinal cords of Lewis rats with experimental autoimmune encephalomyelitis," Brain Research, vol. 1453, pp. 77-86, 2012.

[41] A. Paré, B. Mailhot, S. A. Lévesque, and S. Lacroix, "Involvement of the IL-1 system in experimental autoimmune encephalomyelitis and multiple sclerosis: breaking the vicious cycle between IL-1 $\beta$ and GM-CSF," Brain, Behavior, and Immunity, 2016.

[42] S. A. Lévesque, A. Paré, B. Mailhot et al., "Myeloid cell transmigration across the CNS vasculature triggers IL- $\beta$-driven neuroinflammation during autoimmune encephalomyelitis in mice," The Journal of Experimental Medicine, vol. 213, no. 6, pp. 929-949, 2016.

[43] C. C. Lin, T. R. Bradstreet, E. A. Schwarzkopf et al., "IL1-induced Bhlhe40 identifies pathogenic T helper cells in a model of autoimmune neuroinflammation," The Journal of Experimental Medicine, vol. 213, no. 2, pp. 251-271, 2016.

[44] T. Shin, M. Ahn, and Y. Matsumoto, "Mechanism of experimental autoimmune encephalomyelitis in Lewis rats: recent insights from macrophages," Anatomy \& Cell Biology, vol. 45, no. 3, p. 141, 2012.

[45] T. Shin, M. Ahn, Y. Matsumoto, and C. Moon, "Mechanism of experimental autoimmune neuritis in Lewis rats: the dual role of macrophages," Histology and Histopathology, vol. 28, no. 6, pp. 679-684, 2013.

[46] H. Kim, M. Ahn, S. Choi et al., "Potential role of fibronectin in microglia/macrophage activation following cryoinjury in the rat brain: an immunohistochemical study," Brain Research, vol. 1502, pp. 11-19, 2013.
[47] S. Rossi, T. Lo Giudice, V. De Chiara et al., "Oral fingolimod rescues the functional deficits of synapses in experimental autoimmune encephalomyelitis," British Journal of Pharmacology, vol. 165, no. 4, pp. 861-869, 2012.

[48] I. Stojanovic, S. Vojinovic, S. Ljubisavljevic et al., "INF- $\beta 1 b$ therapy modulates 1 -arginine and nitric oxide metabolism in patients with relapse remittent multiple sclerosis," Journal of the Neurological Sciences, vol. 323, no. 1-2, pp. 187-192, 2012.

[49] A. Stępień, M. Chalimoniuk, N. Lubina-Dąbrowska, S. J. Chrapusta, H. Galbo, and J. Langfort, "Effects of interferon $\beta$-1a and interferon $\beta$-1b monotherapies on selected serum cytokines and nitrite levels in patients with relapsing-remitting multiple sclerosis: a 3-year longitudinal study," NeuroImmunoModulation, vol. 20, no. 4, pp. 213-222, 2013.

[50] K. Rejdak, A. Petzold, Z. Stelmasiak, and G. Giovannoni, "Cerebrospinal fluid brain specific proteins in relation to nitric oxide metabolites during relapse of multiple sclerosis," Multiple Sclerosis, vol. 14, no. 1, pp. 59-66, 2008.

[51] A. I. Danilov, M. Andersson, N. Bavand, N. P. Wiklund, T. Olsson, and L. Brundin, "Nitric oxide metabolite determinations reveal continuous inflammation in multiple sclerosis," Journal of Neuroimmunology, vol. 136, no. 1-2, pp. 112-118, 2003.

[52] K. Rejdak, M. J. Eikelenboom, A. Petzold et al., "CSF nitric oxide metabolites are associated with activity and progression of multiple sclerosis," Neurology, vol. 63, no. 8, pp. 1439-1445, 2004.

[53] O. Ciccarelli, F. Barkhof, B. Bodini et al., "Pathogenesis of multiple sclerosis: insights from molecular and metabolic imaging," The Lancet Neurology, vol. 13, no. 8, pp. 807-822, 2014.

[54] M. Filippi, M. A. Rocca, F. Barkhof et al., "Association between pathological and MRI findings in multiple sclerosis," The Lancet Neurology, vol. 11, no. 4, pp. 349-360, 2012.

[55] M. I. Gaitán, C. D. Shea, I. E. Evangelou et al., "Evolution of the blood-brain barrier in newly forming multiple sclerosis lesions," Annals of Neurology, vol. 70, no. 1, pp. 22-29, 2011.

[56] J. M. Frischer, S. Bramow, A. Dal-Bianco et al., "The relation between inflammation and neurodegeneration in multiple sclerosis brains," Brain, vol. 132, no. 5, pp. 1175-1189, 2009.

[57] L. Haider, "Inflammation, iron, energy failure, and oxidative stress in the pathogenesis of multiple sclerosis," Oxidative Medicine and Cellular Longevity, vol. 2015, Article ID 725370, 10 pages, 2015.

[58] M. Soilu-Hänninen, J. O. Koskinen, M. Laaksonen, A. Hänninen, E.-M. Lilius, and M. Waris, "High sensitivity measurement of CRP and disease progression in multiple sclerosis," Neurology, vol. 65, no. 1, pp. 153-155, 2005.

[59] A. N. Carvalho, O. Firuzi, M. Gama, J. van Horssen, and L. Saso, "Oxidative stress and antioxidants in neurological diseases: is there still hope?” Current Drug Targets, vol. 18, no. 16, pp. 1-14, 2017.

[60] T. Draheim, A. Liessem, M. Scheld et al., "Activation of the astrocytic Nrf2/ARE system ameliorates the formation of demyelinating lesions in a multiple sclerosis animal model," Glia, vol. 64, no. 12, pp. 2219-2230, 2016.

[61] S. Murakami and H. Motohashi, "Recent advances in elucidating KEAP1-NRF2 functions in hematopoietic/immune cells and leukemic cells," Rinsho Ketsueki, vol. 57, no. 10, pp. 1860-1868, 2016.

[62] I. E. Morales Pantoja, C. Hu, N. I. Perrone-Bizzozero, J. Zheng, and O. A. Bizzozero, "Nrf2-dysregulation correlates 
with reduced synthesis and low glutathione levels in experimental autoimmune encephalomyelitis," Journal of Neurochemistry, vol. 139, no. 4, pp. 640-650, 2016.

[63] P. Bozaykut, B. Karademir, B. Yazgan et al., "Effects of vitamin e on peroxisome proliferator-activated receptor $\gamma$ and nuclear factor-erythroid 2-related factor 2 in hypercholesterolemiainduced atherosclerosis," Free Radical Biology and Medicine, vol. 70, pp. 174-181, 2014.

[64] D.-H. Lee, R. Gold, and R. A. Linker, "Mechanisms of oxidative damage in multiple sclerosis and neurodegenerative diseases: therapeutic modulation via fumaric acid esters," International Journal of Molecular Sciences, vol. 13, no. 9, pp. 11783-11803, 2012.

[65] R. W. Orrell, R. J. M. Lane, and M. Ross, "A systematic review of antioxidant treatment for amyotrophic lateral sclerosis/motor neuron disease," Amyotrophic Lateral Sclerosis, vol. 9, no. 4, pp. 195-211, 2008. 


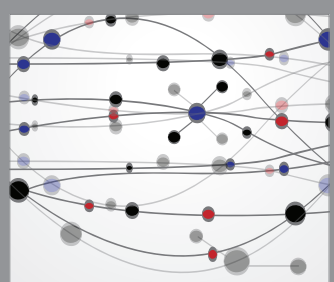

The Scientific World Journal
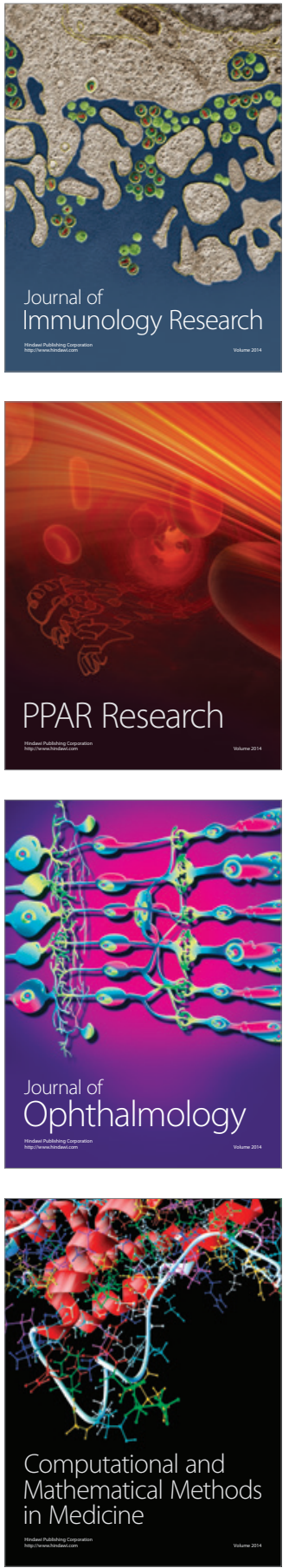

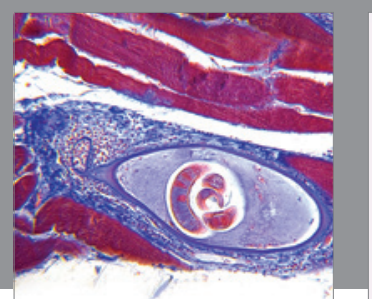

Gastroenterology Research and Practice

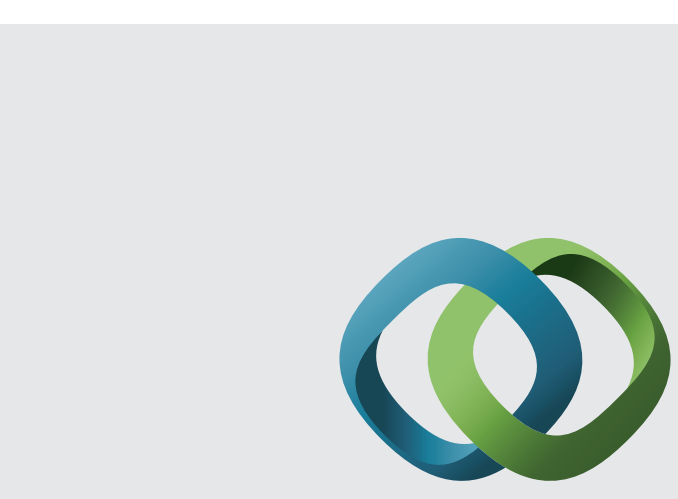

\section{Hindawi}

Submit your manuscripts at

http://www.hindawi.com
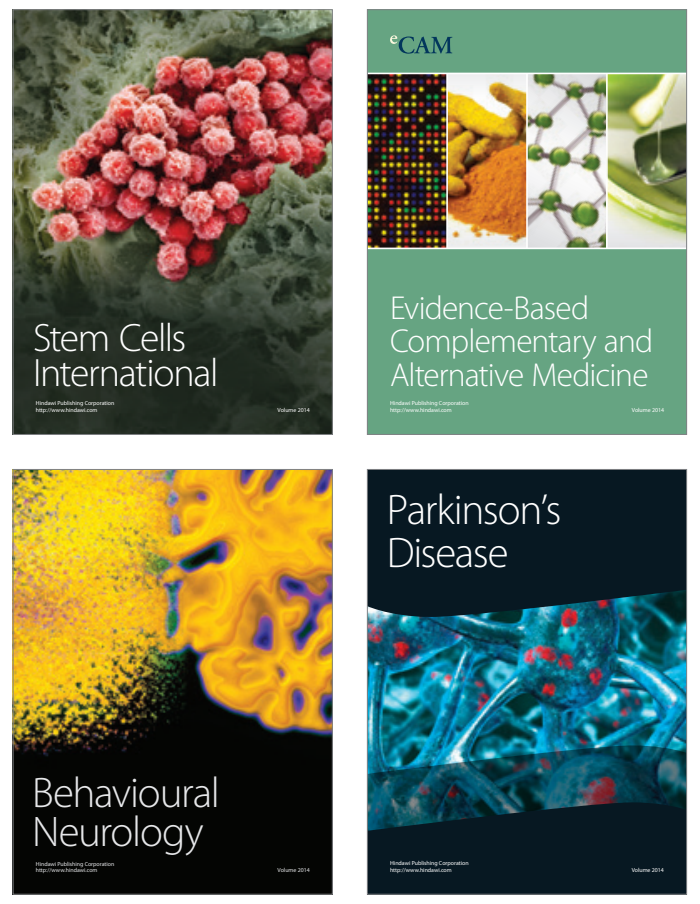
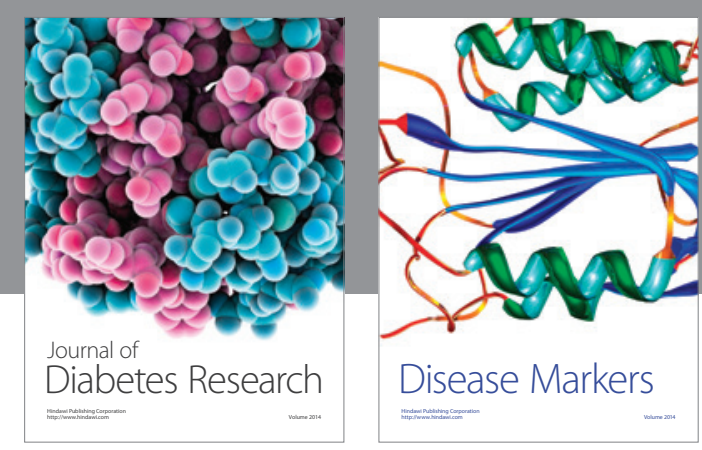

Disease Markers
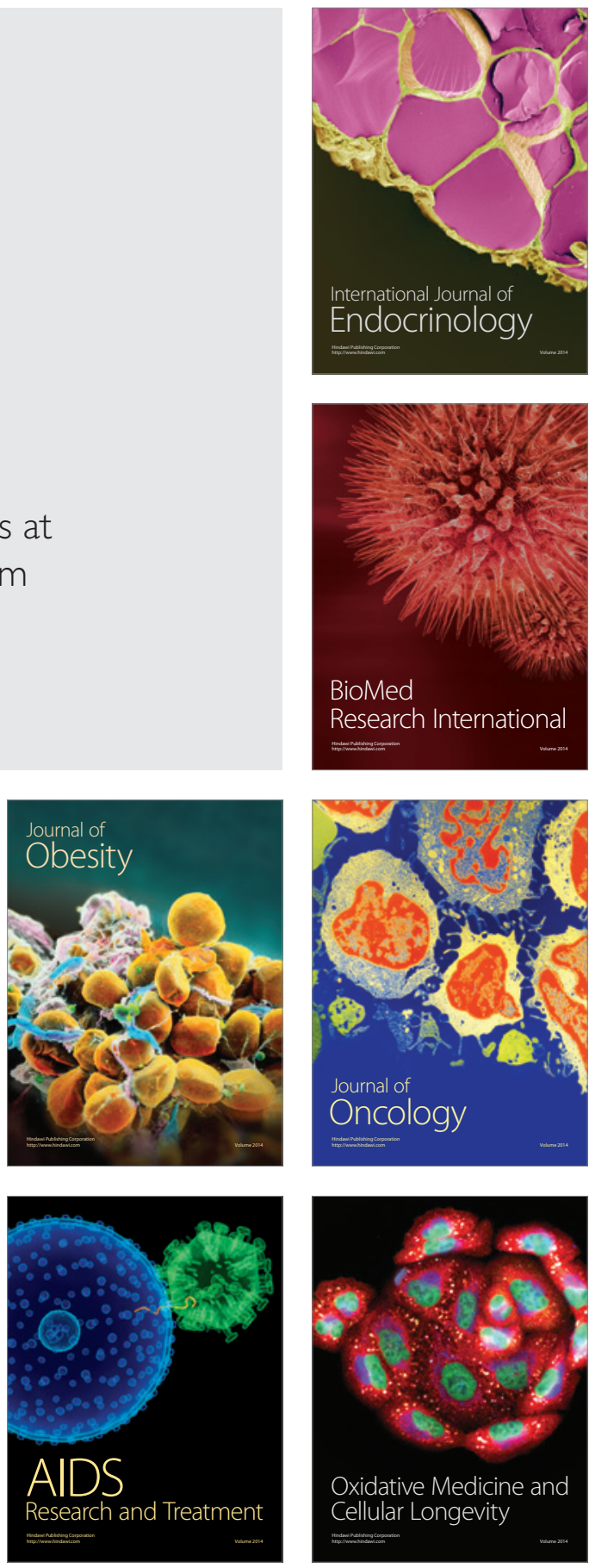